\title{
Königsberg et Strasbourg : une lueur consolatrice dans les ténèbres des temps
}

\section{Klaus Garber}

Traducteur : Jean-Louis Elloy

\section{Q OpenEdition Journals}

Édition électronique

URL : https://journals.openedition.org/rbnu/2805

DOI : $10.4000 /$ rbnu.2805

ISSN : 2679-6104

\section{Éditeur}

Bibliothèque nationale et universitaire de Strasbourg

\section{Édition imprimée}

Date de publication : 1 mai 2012

Pagination : 8-13

ISSN : 2109-2761

\section{Référence électronique}

Klaus Garber, «Königsberg et Strasbourg : une lueur consolatrice dans les ténèbres des temps », La Revue de la BNU [En ligne], 5 | 2012, mis en ligne le 01 mai 2012, consulté le 25 août 2021. URL : http:// journals.openedition.org/rbnu/2805; DOI : https://doi.org/10.4000/rbnu.2805

Ce document a été généré automatiquement le 25 août 2021.

\section{c) (i) (2)}

La Revue de la BNU est mise à disposition selon les termes de la Licence Creative Commons Attribution - Pas d'Utilisation Commerciale - Partage dans les Mêmes Conditions 4.0 International. 


\title{
Königsberg et Strasbourg : une lueur consolatrice dans les ténèbres des temps
}

\author{
Klaus Garber
}

Traduction : Jean-Louis Elloy

\section{NOTE DE L'ÉDITEUR}

Droits réservés - Si vous êtes ayants droits de cet auteur, merci de nous contacter

1 Les livres comme les bibliothèques ont une histoire et ils connaissent, eux aussi, leur lot d'aventures extraordinaires. Mais il ne s'est pas souvent produit qu'au moment où d'importantes bibliothèques de rang européen étaient rayées de l'histoire et sombraient, des bras secourables se soient levés pour accomplir, au milieu de l'horreur, une action salvatrice. C'est une telle histoire que nous voudrions rapporter ici.

2 Le 24 août 1870, la bibliothèque municipale de Strasbourg fut entièrement anéantie. Le Temple Neuf où elle avait été déménagée servit de cible facile à l'armée prussienne lors du bombardement de Strasbourg qui précéda la prise de la ville. Une des plus grandes et des plus riches bibliothèques de l'ancienne aire linguistique allemande avait d'un seul coup été rayée de la carte. Des milliers de manuscrits et imprimés irremplaçables, des sections entières, comme celle consacrée aux alsatiques et, avec elles, des milliers de brochures uniques, de dissertations, de poèmes de circonstance, de feuilles et de tracts, tout cet ensemble de documents collectés pendant plusieurs siècles fut définitivement perdu et ne pouvait pour l'essentiel être remplacé.

3 Ce fut là sans doute la plus grande catastrophe que subit une bibliothèque au cours $d u$ $19^{\mathrm{e}}$ siècle. Elle annonçait déjà cependant la folie destructrice qui allait déferler sur les bibliothèques européennes et particulièrement sur celles d'Allemagne lors de la Seconde Guerre mondiale. Strasbourg connut le sort futur de nombreuses bibliothèques dont la richesse des fonds en livres et en manuscrits était comparable. Parmi d'autres 
exemples, mentionnons simplement la destruction de la bibliothèque municipale de Hambourg et de ses 800000 volumes environ, pendant l'«action Gomorrha » de juillet 1943, ou celle des bibliothèques municipales de Leipzig et de Francfort. Aucune catégorie d'établissements ne fut épargnée: les bibliothèques universitaires, régionales, celles des églises, furent touchées de la même façon. Jamais une catastrophe n'avait atteint une ampleur de cet ordre, par laquelle se manifestait de manière si sévère l'effondrement d'une civilisation.

Le destin des bibliothèques d'Allemagne de l'Est et par-delà ce territoire, celles de l'Europe centrale, mérite d'être rappelé. Partout où les armées allemandes ont fait leur entrée, leur occupation a provoqué un chaos et des ravages très importants. Ces fléaux ont frappé la Pologne et la Russie aussi bien que la Biélorussie et l'Ukraine. Mais l'histoire des bibliothèques de l'Est de l'Allemagne doit être dissociée de cette situation générale. Jusqu'en 1944 elles restèrent préservées de tout dommage. Et les bibliothèques municipales de Breslau, Danzig ou Königsberg ainsi que les bibliothèques universitaires de Breslau et de Königsberg, toutes marquées par une forte empreinte régionale, étaient les dépositaires d'un très riche trésor.

5 Mais leur heure ne devait pas tarder avec l'approche du front russe. On a alors, dans la hâte, procédé à des déménagements, principalement dans les châteaux, les entrepôts, les monastères et les églises des alentours. Toute précaution est restée vaine. La guerre totale s'est abattue aussi bien sur les humains sans défense que sur les nombreux livres privés de leurs protecteurs. De fait, dans les cas de Breslau et de Danzig, les volumes déménagés - et à bien des égards, il s'agissait des plus précieux - ont subi bien plus de dégâts que ceux qui avaient été laissés sur place. Comme par miracle, les deux bibliothèques municipales dans les «forteresses » de Danzig et de Breslau, toutes deux riches d'une longue tradition, ont été exceptionnellement épargnées par la destruction. Elles sont redevenues après la guerre d'importants centres de collecte et d'acquisitions. 


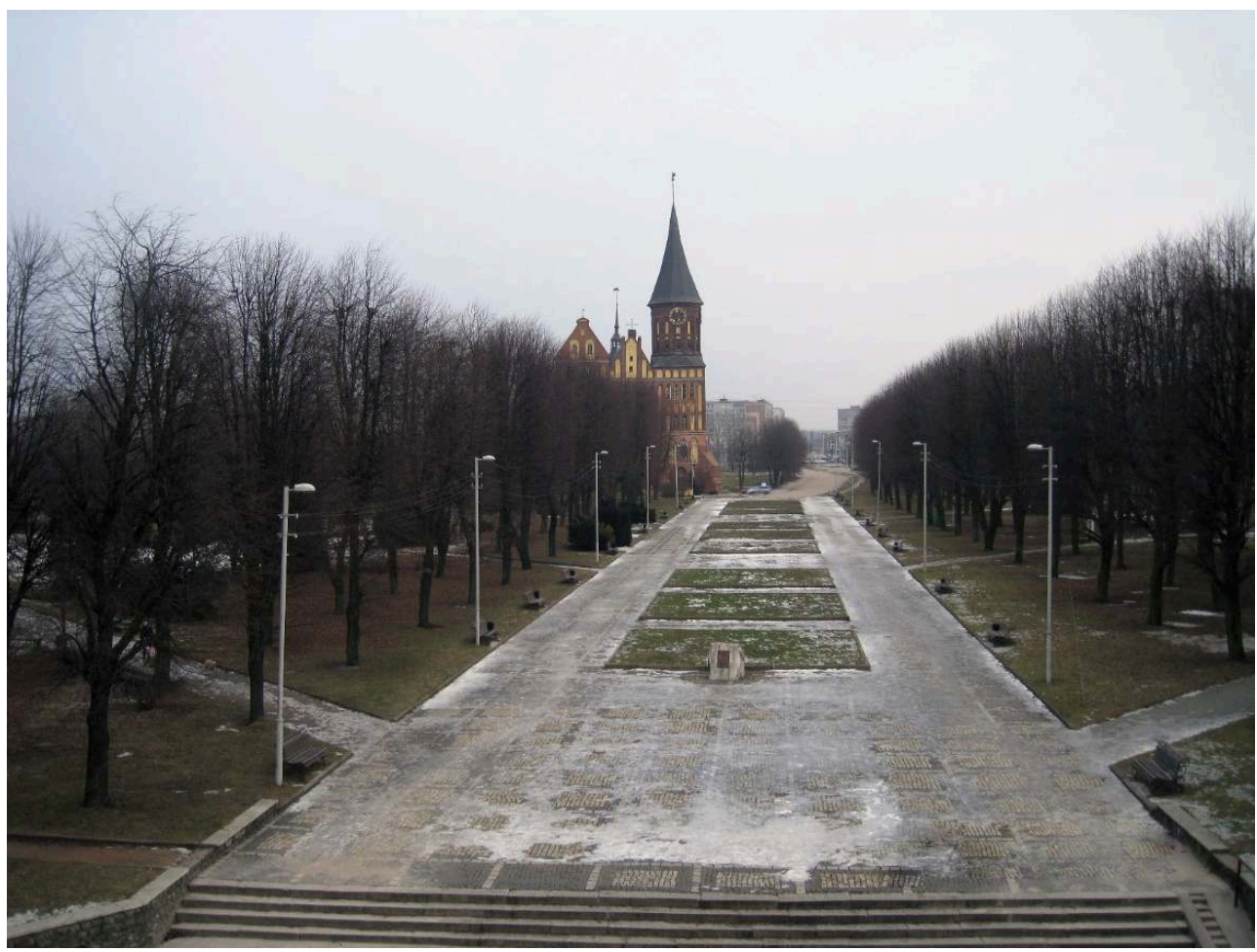

Département de la protection du patrimoine de la région de Kaliningrad

6 Aucune ville cependant n'a autant subi la rage de Némésis que la métropole orientale de l'Empire allemand, Königsberg. L'histoire de ses deux grandes bibliothèques, la municipale et l'universitaire, nous ramène à l'époque de la fondation du duché de Prusse. Le duc Albrecht avait entrepris la construction d'une bibliothèque. Or à cette époque la Réforme faisait son entrée dans la ville et les responsables religieux et municipaux s'étaient très tôt préoccupés de rassembler tous les imprimés en rapport avec le changement de confession et de leur assurer un logement durable. Les livraisons locales provenant de toutes sortes d'endroits affluaient en règle générale vers la bibliothèque municipale. Dans l'ancienne Allemagne, ces établissements étaient à la fois les dépositaires et les initiateurs de l'histoire de la ville. Des générations de collectionneurs y avaient laissé leurs dons. Et aucune de ces nouvelles créations en pleine expansion n'avait dû alors être reconstituée à la suite de pertes ou de destructions.

7 En 1944, à Königsberg également, on procéda à un déménagement des collections. Plus de vingt endroits sur tout le territoire de la Prusse servirent de dépôts pour les livres et les manuscrits. Un an encore avant la fin de la guerre, on ne s'imaginait pas que des troupes étrangères puissent un jour pénétrer dans le pays. L'aveuglement était total. Ce n'est que pour les archives d'État de la Prusse que l'on choisit une autre façon de faire. Dans ce cas, les documents furent en grande partie transférés à l'Ouest et survécurent ainsi à la catastrophe. Mais ce qui était resté dans le pays tomba aux mains des vainqueurs, quand ce ne fut pas détruit ou endommagé à la suite des bombardements, des combats ou des actes de vandalisme. Les Russes, les Polonais, les Lituaniens se partagèrent le butin. Sur place, à Königsberg où se poursuivaient les destructions, il ne subsista pour ainsi dire plus rien. 
8 C'était le début d'une nouvelle histoire. Königsberg était devenue une ville russe. Elle formait une enclave, hermétiquement séparée des territoires environnants. Les informations sur les manuscrits ou les livres qui auraient été épargnés étaient rares. Le monde des spécialistes en conclut, jusque dans les années 1970, que toute culture bibliographique avait disparu de Königsberg. Jusqu'à tout récemment encore, on retrouve çà et là cette appréciation dans des usuels ou des dictionnaires de référence. Ce n'est que lentement que la lumière est apparue. Des historiens allemands qui avaient travaillé à Moscou ou à Leningrad rapportèrent les premiers qu'ils avaient eu entre les mains des livres provenant de Königsberg. De là naquit l'hypothèse que, comme dans les cas de Leipzig, Dresde, Berlin ou encore de Hambourg, Brême et Lübeck, à Königsberg également, les fonds qui avaient été déménagés n'avaient pas été complètement anéantis mais en partie déplacés en Union soviétique.

9 Pour s'en assurer, il fallait mener à bien des voyages qui se consacreraient à cette recherche. L'auteur de ces lignes les a entrepris depuis la fin des années 1970 avec le soutien de la Deutsche Forschungsgemeinschaft (DFG) et s'est systématiquement mis en quête des livres provenant des anciennes bibliothèques allemandes. Un livre intitulé Le livre ancien dans l'ancienne Europe (Das alte Buch im alten Europa) en fait état. Il y est question de Königsberg. Le résultat auquel nous sommes parvenus n'a pas manqué de surprendre: on a conservé bien plus d'anciens et précieux livres et manuscrits de Königsberg qu'on a pu le supposer dans les premières décennies qui ont suivi la fin de la guerre. Il suffisait seulement d'élargir les recherches ; elles ne devaient pas se limiter au territoire de la Russie mais embrasser l'immense continent soviétique - et même parfois conduire au-delà.

10 Nous nous sommes tout d'abord rendus en Pologne. À Breslau, nous avons constaté avec stupéfaction l'immense richesse qu'offre le trésor de la littérature silésienne. Mais nous avons découvert aussi des collections d'ouvrages signés par des poètes de Königsberg, au premier rang desquels ceux de Simon Dach, natif de la ville. Cependant ces trésors provenaient d'un fonds ancien de Breslau, ils n'étaient pas entrés dans l'établissement immédiatement après la guerre. À Varsovie, à la Bibliothèque nationale, la situation était tout autre. La bibliothèque avait été incendiée par les occupants allemands après l'insurrection polonaise de 1944. Après la guerre, il fallut la reconstruire entièrement et reconstituer ses fonds. Les livres des anciennes bibliothèques allemandes se trouvant alors en territoire polonais y contribuèrent efficacement. On fit venir de Silésie et de Poméranie, aussi bien que de Prusse orientale ou occidentale, de précieux ouvrages qui trouvèrent dans la capitale polonaise une nouvelle résidence.

11 Une grande surprise nous y attendait à l'automne 1979: parmi ces ouvrages se trouvaient des fonds renommés de Königsberg. Ils ne provenaient pas, pour la majeure partie d'entre eux, de la Bibliothèque nationale et universitaire mais de la bibliothèque municipale de Königsberg. Jamais jusqu'à ce jour nous n'avions eu la moindre information à leur sujet. Cette dernière bibliothèque avait son siège jusqu'en 1944 dans les pittoresques locaux de l'ancienne université de Königsberg, sur l'île du Kneiphof. Lors de la première attaque aérienne anglaise en août 1944, l'ancienne université avait également été touchée par les bombes. On en avait conclu que la bibliothèque municipale, et avec elle les archives de la ville, avaient été complètement détruites. Mais il s'avérait alors que de riches collections de recueils de brochures, souvent uniques et de grande valeur, étaient parvenus à Varsovie. Cette excellente nouvelle fut 
transmise par nos soins dans un rapport à la DFG. Pour d'autres publications plus détaillées, il était encore trop tôt.

Les découvertes, en effet, ne s'arrêtèrent pas là, et continuèrent tout d'abord en territoire polonais. Dans l'ancienne ville de Thorn (aujourd'hui Toruń) sur la Vistule, que les destructions avaient épargnée, on édifia après la guerre une université qu'il fallut doter d'une bibliothèque. Pour la pourvoir, c'est une nouvelle fois aux sources poméraniennes que l'on s'alimenta principalement. Mais on fit venir aussi de nombreux ouvrages de Königsberg, et cette fois de la Bibliothèque nationale et universitaire, dont de précieux imprimés des $16^{\mathrm{e}}$ et $17^{\mathrm{e}}$ siècles. On pouvait donc nourrir l'espoir de retrouver également des vestiges de la deuxième grande bibliothèque de Königsberg. Il se vit exaucé en Russie où nous nous sommes rendus pour la première fois en 1984 et où nous sommes retournés ensuite régulièrement. Les découvertes se multiplièrent.

Dans la Leningrad d'alors, nous avons visité la Bibliothèque nationale et celle de l'Académie. Dans la première, nous sommes tombés sur d'anciens ouvrages de littérature allemande d'une richesse que jusque-là nous n'avions, en-dehors de l'Allemagne, connue qu'à Londres. L'établissement avait été administré au $19^{\mathrm{e}}$ siècle par des bibliothécaires allemands et ils avaient eu une politique d'acquisition extraordinaire. Dans le flot des milliers d'opuscules, deux lieux d'impression nous sautèrent immédiatement aux yeux: Königsberg et Strasbourg. Il apparaissait clairement à un spécialiste que l'on pouvait trouver en ce lieu un grand nombre de textes qui n'existaient plus ailleurs. Mais ils provenaient d'anciens fonds, ce n'étaient pas des «butins de guerre ». À la bibliothèque de l'Académie, en revanche, dans le département des imprimés anciens, se trouvaient des milliers de livres provenant de Königsberg, qui étaient arrivés là après la guerre. Ils comprenaient, pour notre plus grand bonheur, de nombreux volumes de la plus importante et prestigieuse bibliothèque nobiliaire que le sol prussien ait connue : la bibliothèque de la lignée des Wallenrodt. 


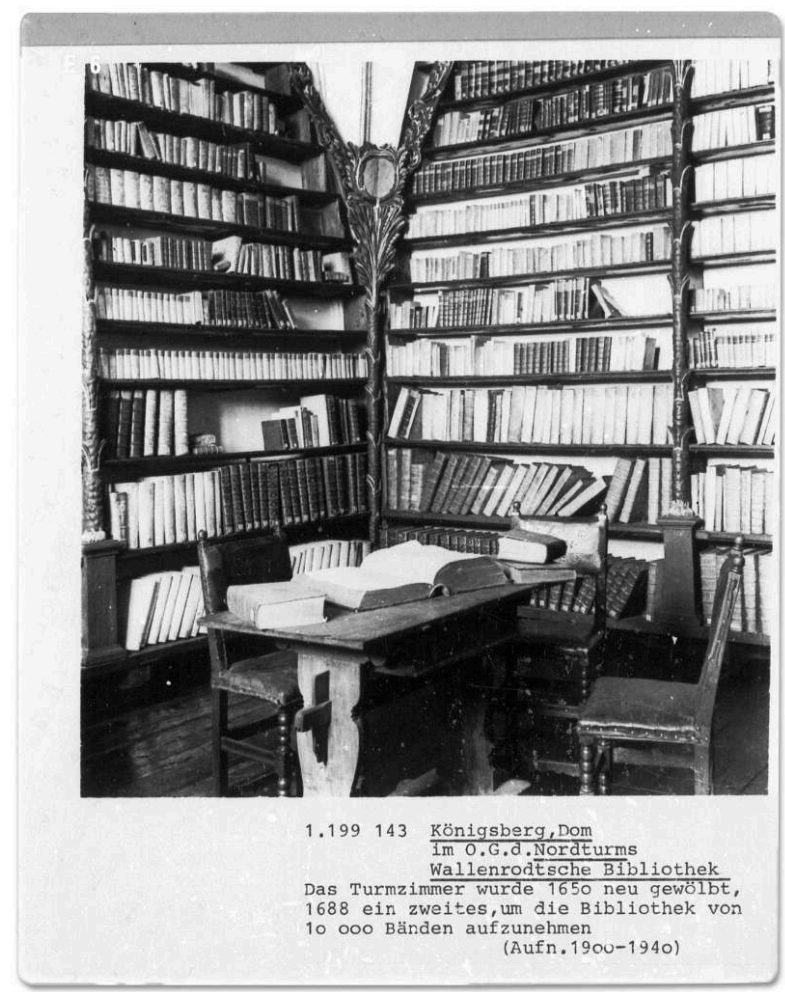

Un des fleurons de la Bibliothèque nationale et universitaire de Königsberg avant 1945, dont une partie se trouvait à la bibliothèque elle-même, et l'autre dans une pièce aménagée dans la tour de la cathédrale.

Département de la protection du patrimoine de la région de Kaliningrad

Peut-on comprendre ce que cela représente pour un chercheur, qui voyage de bibliothèque en bibliothèque, que de trouver en deux endroits tout à fait différents des livres autrefois rassemblés et ensuite séparés, mais qui n'ont pas été perdus ? En 1987, nous avons pu voyager pour la première fois à Vilnius, la capitale de la Lituanie, qui faisait alors partie de l'Union soviétique. Nous venions à ce moment-là de Riga, la capitale lettonne, où nous avions douloureusement déploré la destruction de la splendide bibliothèque municipale lors de l'assaut allemand de 1941. Vilnius nous découvrit aussitôt ses trois bibliothèques: la bibliothèque de l'université, riche d'une longue tradition, et deux créations plus récentes, la Bibliothèque nationale et celle de l'Académie. Nous nous sommes étonnés que ces deux dernières également recèlent de riches fonds de livres de Königsberg. À Vilnius étaient parvenus aussi de précieux Wallenrodtiana. En outre, la ville était devenue après 1945 l'endroit où l'on conservait également les célèbres ouvrages musicaux de Königsberg, connus dans le monde entier, ceux d'un Johann Eckard, d'un Johann Stobaeus et de bien d'autres encore. Il s'y trouvait même des manuscrits en nombre non négligeable.

Varsovie et Toruń en Pologne, Saint-Pétersbourg en Russie et Vilnius en Lituanie sont les quatre villes dans lesquelles on doit avant tout rechercher les imprimés et les manuscrits de Königsberg. On pourrait évoquer bien d'autres lieux: Moscou, Novosibirsk même, Allenstein, Minsk... Mais les quatre premiers mentionnés sont les plus importants. Une vie de chercheur ne suffirait pas à inventorier et à décrire les milliers d'imprimés anciens de Königsberg qui ont été sauvés. À défaut, l'on peut consulter à Osnabrück les microfilms de nombreux ouvrages. Leur catalogage et leur 
numérisation sont en cours. Nous pourrons un jour - espérons qu'il ne se fasse pas trop attendre - récolter les fruits de ce travail. Mais si cela se produit, ce ne sera pas sans compter sur une ville à laquelle on ne s'attendait nullement.

\section{Reconstitution de la bibliothèque Wallenrodt dans la cathédrale de Königsberg}

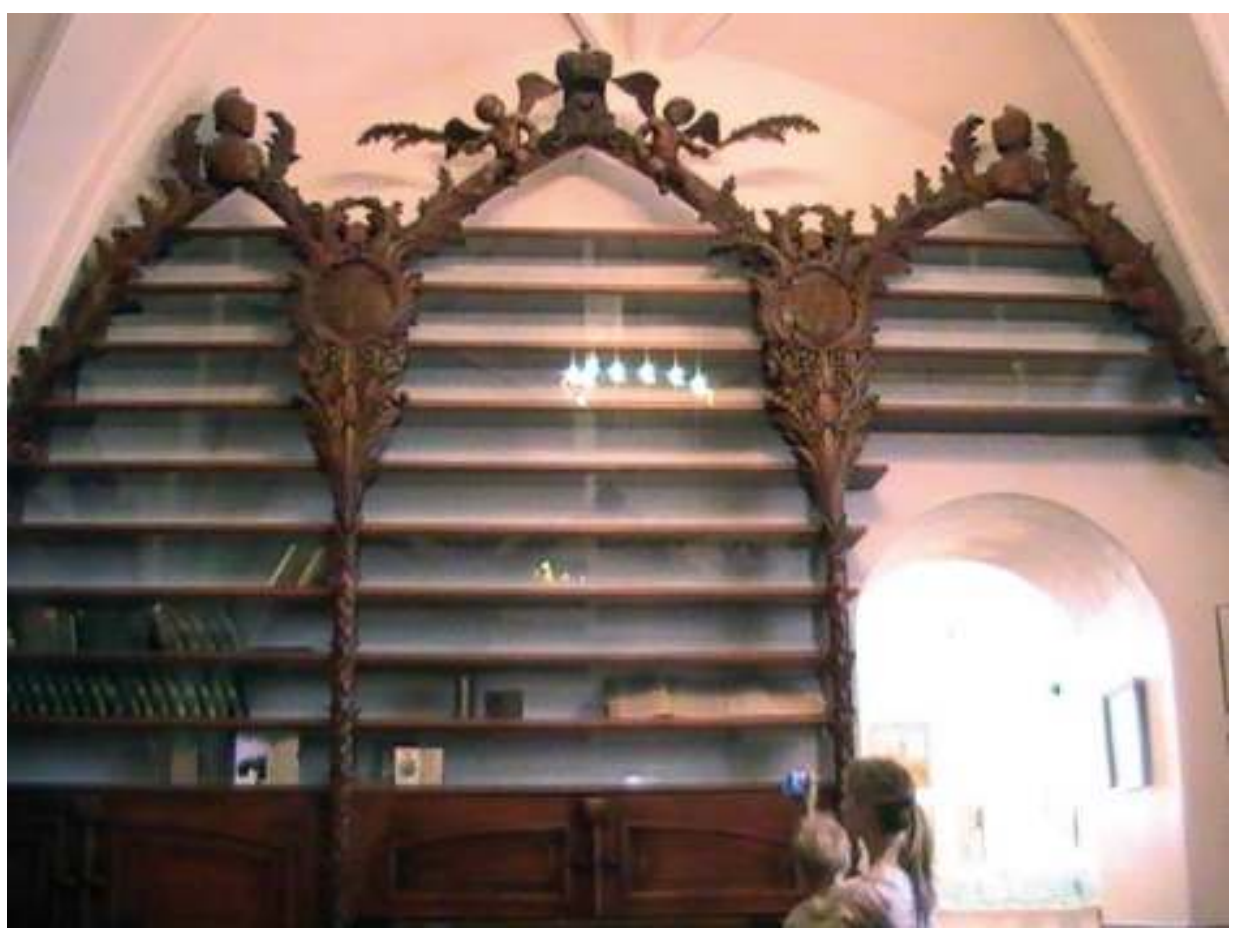

Entreprise en 2005 pour le $750^{\mathrm{e}}$ anniversaire de la ville de Kaliningrad

Département de la protection du patrimoine de la région de Kaliningrad

Et nous voici revenus à notre point de départ, à Strasbourg. Lorsque la catastrophe de 1870 s'est abattue sur la ville, partout dans le monde on a manifesté honte et compassion, tout en faisant preuve de dévouement et d'esprit d'entraide. On lança un appel aux bibliothèques et aux bibliothécaires dans le monde entier, et en premier lieu en Allemagne, pour qu'ils établissent de quels doublons ils pouvaient disposer et pour qu'ils les envoient à Strasbourg. Cet appel eut un écho inespéré, que l'on découvrira dans l'article suivant. Les livres affluèrent de partout dans cette ville qui dans une large mesure avait conservé sa substance historique. Dans le cadre des reconstructions qui à Strasbourg et en Alsace ont été conduites de façon exemplaire (que le nom de Rodolphe Reuss reste toujours dans nos mémoires, lui à qui l'on doit la restauration de la section alsatique de la bibliothèque municipale!), une bibliothèque connut grâce à l'aide extérieure une véritable résurrection, un véritable miracle: l'actuelle Bibliothèque nationale et universitaire de Strasbourg.

Et la lointaine Königsberg participa à ce miracle. Elle envoya tout ce dont elle put se séparer dans la métropole alsacienne, comme Christophe Didier le montre dans son article. C'est ainsi qu'il échoit à Strasbourg d'être aujourd'hui en possession de livres de Königsberg dont les exemplaires qui existaient autrefois là-bas ont été depuis détruits ou perdus. C'est après la Seconde Guerre mondiale que nous avons pu pleinement mesurer le bénéfice dont nous sommes redevables à cet élan donateur d'il y a à peu près cent cinquante ans. Nous ne sommes pas habilités à rendre compte en détail des ouvrages qui ont été sauvés. C'est la tâche des spécialistes qui œuvrent sur place. Mais 
en tant qu'historien du livre ancien de Königsberg, c'est avec émotion et gratitude que nous tournons nos regards vers cette magnifique ville du Rhin supérieur. Le sort qui a frappé toutes les grandes villes d'Allemagne lui a été épargné. Il apparait donc comme une promesse pleine d'avenir que ce soit précisément cette ville, vers laquelle se tourne aujourd'hui l'Europe pleine d'attente, à qui a été dévolue la mission de conserver un trésor sauvé de la destruction de l'ancienne Königsberg.

\section{AUTEURS}

\section{KLAUS GARBER}

Professeur émérite à l'université d'Osnabrück 